\title{
Management of Type 2 Diabetes Mellitus in Older Patients: Current and Emerging Treatment Options
}

Etie Moghissi

To view enhanced content go to www.diabetestherapy-open.com

Received: July 16, 2013 / Published online: October 5, 2013

(C) The Author(s) 2013. This article is published with open access at Springerlink.com

\section{ABSTRACT}

Elderly patients with type 2 diabetes mellitus (T2DM) are a rapidly emerging population that presents unique clinical challenges. This diverse patient group can differ widely in terms of physical and mental status, which can increase their risk of complications including hypoglycemia, falls, and depression. These factors can negatively impact their glycemic control, safety, and quality of life. The risk of hypoglycemic events is elevated among elderly patients with diabetes. In many cases, these events are related to antidiabetic therapy and the pursuit of strict glycemic control. Fear of a hypoglycemic episode, on the part of the patient and/or healthcare provider, is another major

E. Moghissi ( $\square)$

UCLA David Geffen School of Medicine, University of California, 4644 Lincoln Blvd., Suite 409, Marina del Rey, Los Angeles, CA 90292, USA

e-mail: emoghissi@gmail.com

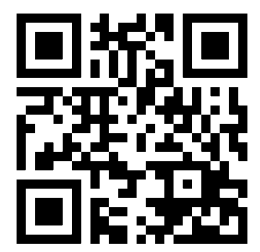

Enhanced content for this article is

available on the journal web site:

www.diabetestherapy-open.com barrier to achieving glycemic control. Hypoglycemic events, even in the absence of awareness of the event (asymptomatic), can have negative consequences. To help manage these risks, several national and international organizations have proposed guidelines to address individualized treatment goals for older adults with diabetes. This article reviews current treatment guidelines for setting glycemic targets in elderly patients with $\mathrm{T} 2 \mathrm{DM}$, and discusses the role of emerging treatment options in this patient population.

Keywords: Elderly; Emerging therapies; Hypoglycemia; Insulin; Management; Treatment guidelines; Type 2 diabetes

\section{INTRODUCTION}

Among persons aged 65 years and older living in the United States, the estimated prevalence of diabetes mellitus ranges from $22 \%$ to $33 \%$ [1]. The number of older individuals with diabetes is expected to grow as the US population ages [1]. By 2050, projections suggest that almost 26.7 million persons aged 
65 years and older living in the United States will be diagnosed with diabetes [2, 3].

Type 2 diabetes mellitus (T2DM) accounts for $90-95 \%$ of diabetes cases in adults [4]. As with younger adults, achieving and maintaining glycemic control is important in elderly patients with T2DM to prevent acute complications of hyperglycemia and reduce the risk of long-term complications [5, 6]. Although some elderly patients will be able to maintain glycemic control with lifestyle modification and oral antidiabetic drugs (OADs), most will eventually require insulin because of the progression of T2DM [7-9].

Management of T2DM in elderly patients is complicated by the clinical and functional heterogeneity of this patient population [6]. Some older patients with T2DM may have developed the disease in middle age and experienced years of comorbidity, whereas others may be newly diagnosed or may have had years of undiagnosed comorbidity or few complications. Older adults also differ with regard to physical robustness, physical and cognitive functioning, health status, and life expectancy. Clinicians who treat elderly patients with T2DM must consider this heterogeneity when setting and prioritizing treatment goals.

Furthermore, older adults with diabetes are more likely than older non-diabetic adults to suffer from geriatric syndromes, including conditions such as polypharmacy, depression, cognitive impairment, urinary incontinence, chronic pain, and injurious falls $[1,5,6]$. It is also important to consider pharmacokinetics when establishing a medication regimen for elderly patients with T2DM [10]. In particular, changes in renal function are common in the elderly and may affect drug metabolism. These and other considerations are listed in Table $1[6$, 11-16].
When managing T2DM in the elderly, it is important to minimize the risk of hypoglycemia. Older patients with diabetes are at a greater risk than are younger patients, even after adjusting for glycemic control [17]. In addition, hypoglycemia presents a higher risk for comorbid events and mortality in elderly patients with diabetes compared with younger patients [1]. Hypoglycemic events in the elderly are independently associated with an increased risk of fall-related fractures and acute cardiovascular events. These events have been shown to adversely impact health-related quality of life as much as, or to an even greater degree than, complications of T2DM [18-20]. Furthermore, evidence suggests that severe hypoglycemic episodes may increase the risk of dementia in patients with T2DM [15]. Conversely, the presence of geriatric syndromes may contribute to the increased risk of hypoglycemia and its complications in elderly patients with T2DM and should be assessed when evaluating hypoglycemia risk (Table 2) $[1,5,17,21-25]$.

The hypoglycemic risk associated with some antidiabetic agents may present the greatest barrier to optimal glycemic control in elderly patients [26]. Therefore, diabetes therapies with the lowest rates of hypoglycemia should be considered for this patient population. Emerging treatment options that may meet this need include both approved and investigational incretin-based therapies and sodium glucose co-transporter 2 (SGLT2) inhibitors, as well as ultra-long-acting insulins that are in late-stage clinical development. The aim of this article is to review current treatment guidelines for setting glycemic targets in elderly patients with T2DM, and to discuss the role of emerging treatment options for this patient population. 
Table 1 Characteristics to consider when individualizing therapy in older patients with type 2 diabetes mellitus (T2DM)

Clinical characteristics Considerations

Comorbid conditions $[6,12,13]$

Younger age at T2DM onset increases cumulative exposure to hyperglycemia and subsequent risk for complications; older age at onset is associated with higher probability of existing comorbidities and shorter life expectancy

Greater disease burden may increase number of medications, potentially leading to confusion, errors, poor adherence, increased adverse effects, costs, drug-drug interactions, and patient frustration

Patients with other medical conditions expected to reduce life expectancy (i.e., shorten the period during which diabetic complications might develop) should be assigned higher HbAlc targets

T2DM duration [12]

Presence of macrovascular (CV) disease $[11,12,14]$

Frailty and ability to self-manage should be addressed and complexity of therapy tailored accordingly

Intensive treatment may be more likely to have benefits the earlier it is begun. This may especially be true in patients with a family history of early CAD

T2DM patients with a history of myocardial infarction are at high risk for recurrent events

Intensive treatment in patients with a prior CV event may not reduce new events or decrease mortality

Higher HbAlc targets may be more appropriate for older patients with a prior CV event

Greater reductions in morbidity and mortality may result from control of CV risk factors than from tight glycemic control in older patients with T2DM

History of severe hypoglycemia $[12,15]$

Dementia is associated with episodes of severe hypoglycemia

Less intensive HbAlc targets are appropriate for patients with recent severe hypoglycemia

Psychological, social, and economic characteristics

Safety concerns and support systems [12]

Adverse effects of medications $[6,12]$

Psychological and cognitive status $[6,12,15]$

Economic considerations $[12,16]$

Quality of life [12]
Highly intensive targets are inappropriate for insulin-treated patients who live alone and have no routine daily check made by family, friends, or neighbors

Patient education and health coaching may have positive effects on patient empowerment, self-care, and outcomes

Insulin or sulfonylureas: possible weight gain, edema, heart failure

Thiazolidinediones: possible fractures

Metformin and certain incretin-based therapies: gastrointestinal adverse effects

Medication reactions increase with polypharmacy. Because intensive glycemic targets require polypharmacy, the risk-benefit ratio of adding other drugs should be carefully balanced with the need to intensify therapy

Depression limits successful goal attainment

Loss of cognitive function may be amplified in older T2DM patients with mild clinical or subclinical cerebrovascular disease or concomitant Alzheimer's disease

Cost of therapy may be prohibitive for patients on a fixed income

Consider treatment with older, less expensive drugs that are still effective, especially if less stringent targets are most appropriate

All-cause mortality rates for T2DM patients are higher among lower socioeconomic groups

T2DM is associated with a 2- to 3-fold higher prevalence of functional disabilities and comorbid conditions, mostly related to $\mathrm{CV}$ disease and obesity

$C A D$ coronary artery disease, $C V$ cardiovascular, $H b A I c$ glycosylated hemoglobin 
Table 2 Geriatric syndromes and additional factors that contribute to hypoglycemia in the elderly [5, 17, 21-25]

\begin{tabular}{ll}
\hline Contributing factors & Considerations \\
\hline Poor cognitive function & Increases risk of severe hypoglycemia in patients with T2DM \\
& Severity of deficits increases the risk \\
& Greater rate of decline increases the risk \\
& Higher risk (vs. younger patients) for hypoglycemia-associated compromised renal \\
function & Altered drug elimination compared with younger patients \\
clearance processes & Rate of insulin clearance from the circulation may decline with age \\
& Hypoglycemia counter-regulation is impaired; less efficient compensatory mechanisms to \\
avoid hypoglycemia & Receiving $\geq 5$ medication classes is associated with severe hypoglycemia \\
In addition to sulfonylureas, angiotensin-converting enzyme inhibitors and non-selective \\
beta-adrenoceptor antagonists can predispose to hypoglycemia
\end{tabular}

Elderly patients with diabetes are at increased risk for geriatric syndromes such as polypharmacy, urinary incontinence, depression, falls, chronic pain, and cognitive impairment $T 2 D M$ type 2 diabetes mellitus

\section{GUIDELINES FOR INDIVIDUALIZATION OF GLYCEMIC TARGETS IN THE ELDERLY}

Over the past several years, various US and international organizations have proposed guidelines for establishing glycemic goals (mainly glycosylated hemoglobin; HbA1c) in patients with T2DM and have addressed the need to individualize these goals in older adults $[1,6,27]$. Although guidelines differ with respect to their stringency and conceptual frameworks, there is a general agreement that the potential benefits of achieving tight glycemic control for the individual should be balanced with the risk of hypoglycemia, given the patient's clinical and functional characteristics.
Current treatment guidelines from the American Diabetes Association (ADA) consider the avoidance of hypoglycemia to be of great importance in setting glycemic goals, particularly in older adults [5]. In addition, the clinical and functional heterogeneity of older adults with T2DM must be considered when establishing and prioritizing treatment goals. Older adults who have significant life expectancy and are active with good cognitive function may be treated using goals developed for younger adults with diabetes (i.e., HbA1c $<7 \%$ ), but it is reasonable to set less intensive glycemic goals (e.g., $<8 \%$ ) for elderly patients with advanced diabetes complications, life-limiting medical comorbidities, or substantial cognitive or functional impairment [5]. The latter group of patients is less likely to benefit from a reduced risk of microvascular 
complications, and more likely to experience serious hypoglycemia-related adverse events.

In 2012, a Consensus Development Conference on Diabetes and Older Adults was convened by the ADA to update diabetes treatment guidelines for older adults and address a variety of issues that may be unique to treating diabetes and common comorbidities in the elderly [1]. The framework for establishing reasonable HbA1c targets utilizes a range based on patient characteristics. Older adults who have few coexisting chronic illnesses and a life expectancy $>10$ years are recommended to have an HbA1c target of $<7.5 \%$. For frail older adults, patients whose life expectancy is $<5$ years, and others in whom the risks of tight glycemic control outweigh the benefits, a reasonable HbA1c goal is $<8.5 \%$. These new guidelines, like the existing ADA guidelines $[5,28]$, acknowledge the clinical and functional heterogeneity of this patient population and replace the more stringent guidelines for improving the care of older adults with diabetes released in 2003 by the California Healthcare Foundation (CHF) and the American Geriatrics Society (AGS) [6]. The benefits and risks of implementing the earlier CHF/AGS guidelines were examined in the setting of a community-based, all-inclusive care program for the elderly [29]. Implementation of the guidelines in frail older patients with diabetes ultimately resulted in dramatic improvements in glycemic control based on HbA1c levels and reductions in overall rates of hypoglycemic episodes. However, the risk of a hypoglycemiarelated visit to the emergency department more than doubled in the early phase of guideline implementation. These findings emphasize the importance of close monitoring for severe hypoglycemia when aiming to achieve glycemic goals in frail elderly patients.

Guidelines from the Department of Veterans Affairs and the Department of Defense propose an explicit risk-stratification approach based on the patient's physiologic age or the presence and severity of microvascular complications and major comorbidities [27]. These guidelines suggest the following targets:

- an HbA1c level of $<7 \%$ for patients with life expectancy >10 years, no major comorbidity and absent/mild microvascular complications;

- an $\mathrm{HbA} 1 \mathrm{c}<8 \%$ for those with life expectancy 5-10 years and moderate comorbidity if microvascular complications are absent, mild, or moderate, or if life expectancy is $>10$ years with moderate microvascular complications;

- an HbA1c of $8-9 \%$ if life expectancy is $<5$ years (major comorbidity) whether microvascular complications are absent, mild, moderate, or advanced [27].

A recent publication that considered the results of clinical trials in older adults with T2DM proposed a framework for individualizing glycemic targets in the outpatient setting [12]. The authors concluded that establishing an individualized goal requires consideration of both clinical characteristics and the psychosocioeconomic setting. The most intensive glycemic control can be targeted in patients who are highly motivated, treatmentadherent and knowledgeable with excellent selfcare capacities and comprehensive support systems. In contrast, the least stringent level of glycemic control (HbA1c 8\%) should be targeted for patients who are less motivated and non-adherent with limited insight, poor self-care capacities, and weak support systems. Table 3 [12] shows the proposed approximate HbA1c targets for older adults with T2DM as determined by clinical characteristics, in the absence of severe hypoglycemia.

The International Association of Gerontology and Geriatrics, the European Diabetes Working 
Table 3 Proposed approximate HbAlc targets for older patients with T2DM based on clinical characteristics (in the absence of severe hypoglycemia) [12]

\begin{tabular}{llll}
\hline Age & $\begin{array}{l}\text { T2DM } \\
\text { duration }\end{array}$ & $\begin{array}{l}\text { Macrovascular and microvascular } \\
\text { complications }\end{array}$ & $\begin{array}{l}\text { Treatment intensity (HbA1c } \\
\text { target) }\end{array}$ \\
\hline$>65-75$ years & Short $^{\mathrm{a}}$ & None and none/early & Less intensive $(\sim 7.0 \%)$ \\
& Long $^{\mathrm{b}}$ & None and none/early & Not intensive $(7.0-8.0 \%)$ \\
& Any & Established and/or advanced & Moderately intensive $(\sim 8.0 \%)^{\mathrm{c}}$ \\
$\begin{array}{l}>75 \text { years or infirm } \\
\text { at any age }\end{array}$ & Any & Any & Moderately intensive $(\sim 8.0 \%)^{\mathrm{c}}$ \\
\hline
\end{tabular}

Reproduced with permission from Ismail-Beigi et al. [12]

HbA1c glycosylated hemoglobin, T2DM type 2 diabetes mellitus

${ }^{a}$ Approximately 5-10 years or less

b Approximately $10-20$ years or more

c Goal is to lessen the risk for hypoglycemia while reducing the risk of severe glycosuria, water and electrolyte loss, infections, and nonketotic hyperosmolar coma

Party for Older People, and the International Task Force of Experts in Diabetes published a position statement in 2012 on the management of diabetes in older adults, explicitly addressing the need to avoid hypoglycemia [30]. In general, an HbA1c target range of 7.0-7.5\% should be set, but a more precise goal based on clinical characteristics may need to be recommended. To reduce the risk of hypoglycemia, no patient should have an on-treatment fasting blood glucose (FBG) level of $<6.0 \mathrm{mmol} / \mathrm{L}(108 \mathrm{mg} /$ $\mathrm{dL}$ ), and blood glucose levels below $5 \mathrm{mmol} / \mathrm{L}$ $(90 \mathrm{mg} / \mathrm{dL})$ should be strictly avoided. Furthermore, glucose-lowering therapy should not be initiated unless the FBG level is consistently $\geq 7 \mathrm{mmol} / \mathrm{L}$ (126 mg/dL) [30].

\section{CURRENT AND EMERGING TREATMENT OPTIONS FOR ELDERLY PATIENTS WITH T2DM}

\section{Incretin-Based Therapies}

The incretins glucagon-like peptide-1 (GLP-1) and glucose-dependent insulinotropic polypeptide (GIP) are peptide hormones that are released into the small intestine following a meal and stimulate insulin release from pancreatic beta cells in a glucose-dependent manner [31]. In addition, GLP-1 suppresses glucagon release from alpha cells. These actions result in an increase in glucose uptake by tissues and a decrease in the release of glucose from the liver. The incretin hormone GLP-1 also delays gastric emptying. Both GLP-1 and GIP are rapidly metabolized by the enzyme dipeptidyl peptidase-4 (DPP-4).

Currently approved incretin-based therapies in the United States include GLP-1 receptor agonists (exenatide and liraglutide) and DPP-4 inhibitors (sitagliptin, linagliptin, saxagliptin, and alogliptin). The GLP-1 receptor agonist lixisenatide is being reviewed by the US Food and Drug Administration (FDA) for the treatment of T2DM [32].

GLP-1 receptor agonists are administered subcutaneously, whereas DPP-4 inhibitors are administered orally [31]. Differences also exist in the efficacy of GLP-1 receptor agonists and DPP-4 inhibitors. A recent meta-analysis of randomized controlled trials comparing 
treatments with placebo or other diabetes medication showed that greater reductions in HbA1c are obtained with GLP-1 receptor agonists than with DPP-4 inhibitors $(\sim 1.0 \%$ vs. 0.6-0.8\%) [33]. Similarly, a recent literature analysis showed that treatment with GLP-1 receptor agonists results in significantly reduced HbA1c levels and bodyweight compared with DPP-4 inhibitors [34]. This is consistent with greater patient satisfaction favoring GLP-1 receptor agonists over DPP-4 inhibitors [34]. Both therapies require dose titration during initiation and are associated with a low risk of hypoglycemia. Overall, incretin-based therapies may be an important treatment option for elderly patients with T2DM $[35,36]$.

Exenatide and liraglutide are approved by the FDA for use as an adjunct to diet and exercise to improve glycemic control in patients with T2DM $[37,38]$. However, neither exenatide nor liraglutide are recommended as first-line therapy for patients with inadequate glycemic control on diet and exercise. An extended-release formulation of exenatide (Bydureon ${ }^{\mathrm{TM}}$; Amylin Pharmaceuticals, San Diego, CA), administered by subcutaneous injection once weekly, also has been approved for the management of T2DM [39]. A post-hoc analysis of pooled data from 16 randomized controlled trials of 12-30 weeks' duration, which included 2,067 patients with T2DM treated with exenatide (10 $\mu$ g twice daily), demonstrated comparable improvements in glycemic control in patients aged $<65$ years $(n=1,613)$ and those aged $\geq 65$ years $(n=454)$; the mean change in $\mathrm{HbA1c}$ was $-0.9 \%$ in younger patients and $-1.0 \%$ in older patients (both, $P<0.0001$ ) [40]. Hypoglycemia was more common among patients taking a concomitant sulfonylurea (age $<65$ years, 27.7\%; $\geq 65$ years, $30.2 \%$ ) than in those who were not receiving such treatment (age $<65$ years, $4.0 \%$; $\geq 65$ years, $1.2 \%)[40]$.
A pooled analysis of six randomized trials compared the effects of liraglutide (1.2 or $1.8 \mathrm{mg}$ ) with placebo in adults with T2DM aged $<65$ years $(n=2,231)$ versus those aged $\geq 65$ years $(n=552)$ [41]. After 26 weeks of treatment, reduction in $\mathrm{HbA1c}$ from baseline was statistically significantly greater for both doses of liraglutide than for placebo in both age groups. For patients $\geq 65$ years, liraglutide changed $\mathrm{HbA} 1 \mathrm{c}$ by $-1.35 \%$ at $1.8 \mathrm{mg}$ and $-1.32 \%$ at $1.2 \mathrm{mg}$ dose compared to placebo $(-0.45 \%)$. For patients $<65$ years, liraglutide changed $\mathrm{HbA1c}$ by $-1.39 \%$ and $-1.31 \%$ at each dose, respectively, whereas patients on placebo only changed by $-0.21 \%$ (all, $P<0.05$ ). Similarly, liraglutide reduced FPG from baseline at both dosages for both age groups compared to placebo (all, $P<0.0001$ ). The proportion of patients reporting minor hypoglycemia, characterized as an event in which the patient had a plasma glucose level of $<56 \mathrm{mg} / \mathrm{dL}$ and did not require assistance to treat, was low (4-15\%) and generally comparable between the two age groups for each dose. Of the six patients who reported major hypoglycemia, characterized as an event during which the patient required assistance with food, glucagon or intravenous glucose to treat, all had received liraglutide $1.8 \mathrm{mg}$ in addition to a sulfonylurea, and all were aged $<65$ years [41].

The safety of exenatide and liraglutide has been called into question, and both agents have black box warnings (BBWs) as part of their labeling. Exenatide has a BBW for medullary thyroid cancer (MTC) and a Risk Evaluation and Mitigation Strategy is ongoing to properly evaluate the benefit of the drug compared with the risk of MTC and acute pancreatitis. The FDA have also requested an additional study to assess the risk of other adverse events (e.g., pancreatic cancer, renal disorders, and serious hypoglycemia) [42]. Liraglutide also has 
a BBW for risk of thyroid C-cell tumors, including MTC, and is contraindicated in patients with a history of MTC and in patients with multiple endocrine neoplasia syndrome type 2 [38].

The efficacy and safety of the experimental GLP-1 agonist lixisenatide ( $20 \mathrm{mg} /$ day) in 379 patients aged $\geq 65$ years were evaluated in an analysis of data from six randomized, placebocontrolled, phase 3 trials [32]. In all studies, comparable decreases in HbA1c levels were observed in patients aged $<65$ years and older patients, and significantly greater decreases in HbA1c levels were obtained with lixisenatide versus placebo in both age groups. The incidence of symptomatic hypoglycemia varied depending on background treatment and usually was comparable between the lixisenatide and placebo groups; no relevant differences were observed between younger patients and those aged $\geq 65$ or $\geq 75$ years [32].

The DPP-4 inhibitors sitagliptin, linagliptin, saxagliptin, and alogliptin are FDA-approved as an adjunct to diet and exercise, for use as monotherapy or in combination therapy, in adults with T2DM [43-46]. Data published in presentation abstract form are available from a study of linagliptin in elderly patients and from analyses of data in elderly patients participating in clinical trials of sitagliptin or saxagliptin [47-50].

A 24-week, phase 3, randomized, placebocontrolled trial evaluated linagliptin $5 \mathrm{mg}$ /day in 241 patients (aged $\geq 70$ years) with T2DM inadequately controlled despite metformin, sulfonylurea, and/or insulin therapy [49]. From placebo, the mean change in $\mathrm{HbA1c}$ level with linagliptin at 24 weeks was $-0.64 \%$ $(P<0.0001)$. Although hypoglycemia was more common in the linagliptin group than in the placebo group ( $24.1 \%$ vs. $16.5 \%$, respectively), the difference was not statistically significant.
Hypoglycemic events occurred predominantly in patients receiving concomitant insulin and/ or sulfonylurea therapy. These findings suggest that adjunctive linagliptin is effective in elderly patients with inadequately controlled T2DM and does not impose an excess risk of hypoglycemia. However, when linagliptin is used in combination with insulin or a sulfonylurea, it may be necessary to lower the insulin or sulfonylurea dose to reduce the risk of hypoglycemia.

A 24-week, randomized, double-blind, placebo-controlled, parallel-group study evaluated sitagliptin monotherapy in 206 elderly patients with T2DM [51]. Patients received sitagliptin 50 or $100 \mathrm{mg}$ (depending on renal function) or placebo. The mean age of patients was 72 years and mean baseline HbA1c was $7.8 \%$. The between-group difference (sitagliptin - placebo) of least-squares mean change in HbA1c level for patients with a baseline $\mathrm{HbA} 1 \mathrm{c} \geq 9.0 \%$ was $-1.6 \%(P=0.043)$. Mean average blood glucose levels decreased rapidly once the treatment commenced, with significant improvements observed as early as day 3 of treatment. No incidences of hypoglycemia were reported, and the drug was well tolerated in this population of older patients [51].

A post-hoc analysis of pooled data from three randomized, double-blind studies of 373 elderly (aged $\geq 65$ years) patients compared the effects of sitagliptin versus glipizide or glimepiride added to metformin or diet alone [47]. At 30 weeks, both HbA1c and FBG levels had decreased in both the sitagliptin and sulfonylurea treatment groups, with no statistically significant differences between treatment modalities. However, the incidence of symptomatic hypoglycemia (i.e., that reported as an adverse event) among elderly patients was significantly lower in the 
sitagliptin group than in the sulfonylurea group (6.2\% vs. $28.2 \% ; P<0.001)$.

A once-daily formulation of a sitagliptin/ metformin combination (Janumet $\mathrm{XR}^{\mathrm{TM}}$; Merck \& Co., Inc, Whitehouse Station, NJ, USA) was approved by the FDA in 2012 for the management of patients with T2DM [52]. This combination has been shown to be bodyweight neutral and has an acceptable risk of hypoglycemia; its once-daily dosing may lower the 'pill burden' —an important factor especially for elderly patients [53]. However, it has a BBW for lactic acidosis in the labeling. Metformincontaining medications should be used cautiously in patients with hepatic disease and those aged $>80$ years [53]. In addition, patients taking sitagliptin alone or in combination should be monitored for the development of pancreatitis [53].

A sub-analysis compared the efficacy and safety of saxagliptin in patients aged $<65$ years $(n=351)$ with those aged $\geq 65$ years $(n=104)$ who had participated in a placebo-controlled trial of adjunctive saxagliptin for T2DM inadequately controlled with insulin alone or insulin plus metformin [48]. At 24 weeks, improvements in HbA1c, FBG, and postprandial glucose levels were similar for both age groups, with no statistically significant interactions of treatment by age. The incidence of hypoglycemia was not reported, and no elderly patients discontinued the study because of an adverse event [48].

In January 2013, the DPP-4 inhibitor alogliptin received FDA approval for the treatment of T2DM [46]. It is available in three different formulations: as monotherapy (Nesina $^{\mathrm{TM}}$; Takeda Pharmaceuticals America, Inc, Deerfield, IL), as a fixed-dose combination with metformin $\left(K^{2}{ }^{\mathrm{TM}}{ }^{\mathrm{TM}}\right.$; $\quad$ Takeda Pharmaceuticals America, Inc, Deerfield, IL), and as a fixed-dose combination with pioglitazone (Oseni ${ }^{\mathrm{TM}}$; Takeda Pharmaceuticals America, Inc, Deerfield, IL). Oseni carries a BBW for heart failure due to the pioglitazone component, and Kazano will have a BBW for lactic acidosis due to the metformin component [46].

The study of linagliptin and analyses of other incretin-based therapies in older adults suggest that elderly patients can benefit from these agents at least as much as younger patients do, without a substantial risk of hypoglycemia [4750, 54]. Phase 3 trials in elderly patients with T2DM are ongoing or completed for sitagliptin [55], saxagliptin [56], linagliptin [57], and alogliptin [58]. The results of these studies will provide additional insight on the risks and benefits of incretin therapies in this patient population. It should be noted that GLP-1 receptor agonists have been associated with an increased risk of gastrointestinal adverse effects (e.g., nausea and vomiting), while DPP-4 inhibitors pose an increased risk of nasopharyngitis, urinary tract infection, and headache [59]. GLP-1 receptor agonists also are associated with mild weight loss, whereas DPP-4 inhibitors are weight-neutral [59]. Requirements for dosage adjustment in patients with renal impairment also differ among the incretin-based therapies; however, dose adjustment is not required for patients who have mild hepatic impairment [37-39, 43$45,60-63]$. Before incorporating incretin-based therapies into an individualized regimen for the elderly patient, differences in efficacy and tolerability should be considered in relation to the patient's health status, comorbidities, and concomitant medications.

\section{SGLT2 Inhibitors}

SGLT2 inhibitors comprise an emerging, novel class of antihyperglycemic drugs that inhibit 
glucose reabsorption in the kidney and increase excretion of glucose in the urine [64, 65]. This new, orally administered treatment strategy directly lowers plasma glucose concentration in an insulin-independent manner with a low risk of hypoglycemia. A common adverse effect of SGLT2 inhibitors is an increased incidence of urinary tract and genital infections. Thus, special caution is warranted in elderly patients at higher risk of such infections, e.g., those with urinary incontinence [66].

The SGLT2 inhibitor canagliflozin was recently approved in the United States by the FDA for the treatment of T2DM [67]. Another SGLT2 inhibitor, dapagliflozin, has been approved in the European Union but not in the United States [67]. Additional SGLT2 inhibitors are currently under review by the FDA.

A 102-week, randomized, double-blind, placebo-controlled, phase 3 study evaluated dapagliflozin $(2.5,5$, or $10 \mathrm{mg})$ or placebo added to metformin in 546 patients with T2DM [68]. The mean change from baseline in HbA1c level was $+0.02 \%$ for placebo compared with $-0.48 \%(P=0.0008),-0.58 \%(P<0.0001)$, and $-0.78 \%(P<0.0001)$ for dapagliflozin $2.5,5$, and $10 \mathrm{mg}$, respectively. All dapagliflozin groups had sustained reductions in fasting plasma glucose (FPG) and bodyweight. Recorded hypoglycemic events were infrequent and not severe in nature. An increase in reported urinary tract infections and genital infections was observed for all dapagliflozin groups compared with placebo.

Clinical trials showed that canagliflozin also improves glycemic control, reduces HbA1c, and decreases bodyweight and systolic blood pressure. A 52-week, randomized, doubleblind, phase 3 trial in adults with T2DM compared the effects of canagliflozin $100 \mathrm{mg}$ $(n=483)$ or canagliflozin $300 \mathrm{mg}(n=485)$ with glimepiride $(n=482)$ [69]. After 52 weeks of treatment, reduction in $\mathrm{Hb} 1 \mathrm{Ac}$ from baseline changed $-0.82 \%$ at $100 \mathrm{mg}$ and $-0.93 \%$ at $300 \mathrm{mg}$. The 300-mg canagliflozin dose was statistically significantly superior versus glimepiride $(-0.12 \%$ [95\% CI -0.22 to -0.02 ] Canagliflozin was generally well tolerated; however, both men and women who received canagliflozin reported a greater number of genital mycotic and urinary tract infections. Genital mycotic infections were reported by $7 \%$ of men and $11 \%$ of women taking canagliflozin $100 \mathrm{mg}$ compared to $1 \%$ of men and $2 \%$ of women taking glimepiride. At the $300 \mathrm{mg}$ dose of canagliflozin, $8 \%$ of men and $14 \%$ of women reported this adverse event.

Notably, a 26-week, randomized, doubleblind, placebo-controlled, phase 3 clinical trial investigated the use of canagliflozin in 716 adults aged 55-80 years with T2DM [70]. Results demonstrated that canagliflozin was effective and well tolerated in older patients receiving background therapy with other antidiabetic agents. Treatment with canagliflozin 100 and $300 \mathrm{mg}$ reduced HbA1c levels at 26 weeks compared with placebo $(-0.60 \%,-0.73 \%$, and $-0.03 \%$, respectively; $P<0.001)$. Additionally, treatment with canagliflozin at either dosage reduced bodyweight, FPG, and systolic blood pressure compared with placebo. However, both canagliflozin doses were associated with higher rates of urinary tract infections, genital mycotic infections, and documented hypoglycemia rates compared with placebo. For patients taking canagliflozin $100 \mathrm{mg}$, genital mycotic infections were reported by $3.2 \%$ of men and $15.4 \%$ of women compared to $0 \%$ of men and $2.1 \%$ of women in the placebo group. At the $300 \mathrm{mg}$ dose of canagliflozin, this adverse event was reported by $6.2 \%$ of men and $11.2 \%$ of women. 


\section{Basal Insulins}

Of the currently approved antidiabetic treatments, insulin is the most effective when properly dosed, but there has been a general trend toward under-utilization of insulin across all age groups, including the elderly [71-73]. Recent findings suggest that patients aged $\geq 70$ years can achieve the same level of glycemic control from insulin as do younger patients [74].

In general, insulin analogs are preferred over human insulin because they have a more consistent pharmacokinetic profile and are associated with lower rates of hypoglycemia [75]. A recent pooled analysis of data from five randomized controlled trials, involving a total of 2,695 patients with inadequate control of T2DM on OADs alone, suggests that the addition of insulin glargine results in modestly greater improvements in HbA1c $(P<0.001)$ and FBG $(P<0.001)$ levels compared with NPH insulin in patients aged $\geq 65$ years, whereas in younger patients ( $<65$ years) similar glycemic improvements were observed with the two types of insulin [76]. Overall, among both insulin groups (glargine and $\mathrm{NPH}$ ), the rate of nocturnal symptomatic hypoglycemia, but not daytime (symptomatic or severe) or overall severe hypoglycemia, was slightly but significantly $(P<0.05)$ higher for older adults compared with younger adults [76]. Although patients aged $<65$ years had significantly lower rates of nocturnal symptomatic and severe hypoglycemia with insulin glargine versus $\mathrm{NPH}$ insulin, there were no between-treatment differences in hypoglycemia rates among patients $\geq 65$ years of age [76].

A pooled analysis of three randomized, openlabel phase 3 trials of 22-26 weeks' duration $(N=1,296)$ compared insulin detemir with $\mathrm{NPH}$ insulin in younger ( $<65$ years) and older
( $\geq 65$ years) adults with inadequately controlled T2DM [77]. Patients received basal insulin once or twice daily in combination with either bolus insulin or an OAD, depending on the study. Mean changes in HbA1c and FPG levels were similar for the two treatment groups and for both age groups. The relative risk of overall hypoglycemia was significantly lower with insulin detemir than with NPH insulin for both age groups. However, the relative risk of nocturnal hypoglycemia was statistically significantly lower with insulin detemir in patients aged $<65$ years, but not in those older than 65 years [77].

Ultra-long-acting basal insulins in clinical development may present an even lower risk of hypoglycemia than currently available basal insulin analogs, which would be especially advantageous in elderly patients. Two such insulins currently in development are insulin degludec and LY2605541. Insulin degludec incorporates structural modifications that allow it to form soluble and stable multi-hexamers [7880]. Gradual separation of the monomers from the multi-hexamers following subcutaneous injection results in a protracted and continuous delivery of insulin degludec into the circulation. Insulin degludec demonstrates a flat and stable glucose-lowering effect with once-daily administration [78]. LY2605541, the other investigational basal insulin analog, is a derivative of insulin lispro that contains a single polyethylene glycol (PEG) moiety [79, 81]. The large hydrodynamic size of the analog enabled by the PEG moiety is believed to slow both renal clearance and absorption from the subcutaneous depot. LY2605541 exhibits low intra-subject variability and has a longer serum concentration-time profile and duration of action than insulin glargine [81]. The smoother pharmacokinetic and pharmacodynamic profiles of these novel ultra-long-acting insulins may 
reduce the frequency and magnitude of blood glucose troughs, thereby reducing the frequency and severity of hypoglycemic episodes $[79,81]$.

Results of randomized, open-label, phase 3, treat-to-target trials and of a meta-analysis of patients with T2DM suggest that insulin degludec achieves glycemic control that is comparable to, or better than, that of insulin glargine, with significantly lower rates of overall confirmed and/or nocturnal confirmed hypoglycemia [82-84]. A meta-analysis of seven randomized, open-label, phase 3 trials of insulin degludec in patients aged $\geq 65$ years with type 1 diabetes or T2DM found that rates of nocturnal hypoglycemia, but not overall confirmed hypoglycemia, were significantly lower (by 35\%) in patients treated with insulin degludec versus insulin glargine [85]. Similarly, a phase 2 study in patients with T2DM demonstrated comparable glycemic control with LY2605541 and insulin glargine, but significantly lower rates of nocturnal hypoglycemia (after adjusting for baseline hypoglycemia) with LY2605541 [86]. Overall, studies of insulin degludec and LY2605541 in patients with T2DM suggest that these ultralong-acting formulations of insulin may allow patients to achieve glycemic goals with a reduced risk of hypoglycemia. Moreover, the reduced risk of hypoglycemia may encourage patients and physicians to target tighter glycemic control. Both of these insulin formulations were well tolerated and may represent an important treatment option for elderly patients with T2DM $[82,84]$.

\section{CLINICAL CONSIDERATIONS}

Attaining strict glycemic targets are associated with an increased risk of hypoglycemic events [87]. The occurrence of hypoglycemia is linked with increased morbidity, mortality, and cost, and minimizing hypoglycemia is thought to increase adherence and satisfaction with treatment strategies [88, 89]. Hypoglycemia and fear of hypoglycemia in patients and healthcare providers constitute a major impediment to achieving appropriate glycemic targets, especially in elderly patients. Conversely, failure to recognize hypoglycemia may lead to potentially catastrophic consequences. Therefore, the prevention of hypoglycemia is an important goal in considering glycemic targets and treatment strategies for individuals with T2DM. In keeping with this, professional organizations emphasize safety and avoidance of hypoglycemia in their considerations for choosing regimens and therapeutic agents.

Hypoglycemia remains a key barrier to glycemic control in elderly patients with T2DM. The presence of predisposing factors to hypoglycemia, such as cognitive impairment and renal impairment, must be considered when setting glycemic goals and individualizing therapy in the elderly $[12,15$, 21]. In addition, numerous studies indicate a direct correlation between hypoglycemia and impaired cognitive function, which may interfere with necessary self-care activities required for managing blood glucose levels. A recent study of 563 older adults demonstrated that the executive function domain of cognition was significantly associated with HbA1c levels [90]. Improvement by one unit in executive function directly correlated to a 0.23 decrease in HbA1c $(P=0.02)$. Tasci et al. [91] conducted a prospective and observational study in a small group of elderly individuals with T2DM $(n=10)$ who began treatment with vildagliptin $50 \mathrm{mg}$ twice daily as an adjunct to metformin. After a mean follow-up of 11 months, no significant changes in clinical 
cognitive function were found [91]. The impact of diabetes treatment regimens on cognitive performance in 3,421 elderly patients without dementia was investigated [92]. As expected, older adults with diabetes exhibited significantly diminished performance in many areas of cognitive functions compared with non-diabetic patients, with diabetic patients demonstrating a lower overall composite score for the summary measure of cognitive functioning $(P=0.01)$. However, there were no significant differences in composite scores between elderly patients with diabetes who were being treated with insulin and/or hypoglycemic medications and those who were untreated [92].

Given the current state of knowledge, avoidance of hypoglycemic events is an important consideration in choosing a therapeutic regimen in elderly individuals with T2DM. Metformin constitutes the first line of therapy, and its use is rarely associated with hypoglycemic events [93, 94]. When adequate glucose control necessitates treatment therapies in combination with or as an alternative to metformin, use of a DPP-4 inhibitor, GLP-1 receptor agonist, SGLT2 inhibitor, or insulin analog may be preferred over sulfonylureas or traditional insulins if hypoglycemia is a significant concern in a particular patient. Thiazolidinediones represent an additional class of antidiabetic agents that increase insulin sensitivity and are associated with a low risk of hypoglycemia [95]. Emerging therapies may offer effective and welltolerated treatment alternatives for elderly patients with T2DM. Studies with incretin therapies suggest that elderly patients may achieve similar glycemic benefits to younger patients with a low risk of hypoglycemia [35]. The long-term safety of DPP-4 inhibitors and GLP-1 receptor agonists is under investigation.
The FDA requirement that evidence of cardiovascular safety be provided for new glucose-lowering agents means that all medications developed to enhance incretin action must be evaluated in long-term clinical trials [96]. The forthcoming results of these long-term studies should provide more conclusive information about the safety of GLP-1 receptor agonists, DPP-4 inhibitors, and SGLT2 inhibitors in elderly patients with diabetes.

\section{CONCLUSION}

The population of elderly patients with T2DM in the United States is growing at an alarming rate. Management of T2DM in elderly patients is complicated, given their clinical and functional diversity and the frequent presence of geriatric syndromes, such as polypharmacy, depression, and cognitive impairment.

Insulin remains the most effective treatment for T2DM in elderly patients and can be titrated to decrease any level of HbA1c to, or near, the therapeutic goal. Although rates of severe hypoglycemia are relatively low when basal insulin analogs are used, reason for special caution is required when these agents are used concomitantly with sulfonylureas or in patients at an increased risk of hypoglycemia. Ultralong-acting formulations of insulin in clinical development, including insulin degludec and LY2605541, appear to provide comparable or improved glycemic control with a reduced risk of hypoglycemia compared with insulin glargine.

\section{ACKNOWLEDGMENTS}

The author acknowledges Eric Berlin and The Medicine Group for editorial assistance in the 
development of this manuscript; processing charges and funding for this manuscript were provided by Novo Nordisk.

Dr. Moghissi is the guarantor for this article, and takes responsibility for the integrity of the work as a whole.

Conflict of interest. The author has served as an advisor for Novo Nordisk and Sanofi, and has been a speaker for BI/Lilly, Johnson \& Johnson, and Novo Nordisk.

Open Access. This article is distributed under the terms of the Creative Commons Attribution Noncommercial License which permits any noncommercial use, distribution, and reproduction in any medium, provided the original author(s) and the source are credited.

\section{REFERENCES}

1. Kirkman MS, Briscoe VJ, Clark N, et al. Diabetes in older adults. Diabetes Care. 2012;35:2650-64.

2. Caspersen CJ, Thomas GD, Boseman LA, et al. Aging, diabetes, and the public health system in the United States. Am J Public Health. 2012;102: 1482-97.

3. Narayan KM, Boyle JP, Geiss LS, et al. Impact of recent increase in incidence on future diabetes burden: U.S., 2005-2050. Diabetes Care. 2006;29:2114-6.

4. National diabetes fact sheet: national estimates and general information on diabetes and prediabetes in the United States, 2011. Atlanta, Georgia: U.S. Department of Health and Human Services, Centers for Disease Control and Prevention; 2011.

5. American Diabetes Association. Standards of medical care in diabetes-2012. Diabetes Care. 2012;35(Suppl 1):S11-63.

6. Brown AF, Mangione CM, Saliba D, et al. Guidelines for improving the care of the older person with diabetes mellitus. J Am Geriatr Soc. 2003;51: S265-80.

7. Spain M, Edlund BJ. Introducing insulin into diabetes management: transition strategies for older adults. J Gerontol Nurs. 2011;37:10-5.
8. Fu AZ, Qiu Y, Davies MJ, et al. Initial sulfonylurea use and subsequent insulin therapy in older subjects with type 2 diabetes mellitus. Diabetes Ther. 2012;3:12.

9. Chang AM, Halter JB. Aging and insulin secretion. Am J Physiol Endocrinol Metab. 2003;284:E7-12.

10. Neumiller JJ, Setter SM. Pharmacologic management of the older patient with type 2 diabetes mellitus. Am J Geriatr Pharmacother. $2009 ; 7: 324-42$.

11. Gerstein HC, Miller ME, Byington RP, et al. Effects of intensive glucose lowering in type 2 diabetes. N Engl J Med. 2008;358:2545-59.

12. Ismail-Beigi $F$, Moghissi $E$, Tiktin $M$, et al. Individualizing glycemic targets in type 2 diabetes mellitus: implications of recent clinical trials. Ann Intern Med. 2011;154:554-9.

13. Kalyani RR, Saudek CD, Brancati FL, et al. Association of diabetes, comorbidities, and A1C with functional disability in older adults: results from the National Health and Nutrition Examination Survey (NHANES), 1999-2006. Diabetes Care. 2010;33:1055-60.

14. Patel A, MacMahon S, Chalmers J, et al. Intensive blood glucose control and vascular outcomes in patients with type 2 diabetes. $\mathrm{N}$ Engl J Med. 2008;358:2560-72.

15. Whitmer RA, Karter AJ, Yaffe K, et al. Hypoglycemic episodes and risk of dementia in older patients with type 2 diabetes mellitus. JAMA. 2009;301:1565-72.

16. Lipscombe LL, Austin PC, Manuel DG, et al. Income-related differences in mortality among people with diabetes mellitus. CMAJ. 2010;182:E1-17.

17. Bramlage $\mathrm{P}$, Gitt $\mathrm{AK}, \mathrm{Binz} \mathrm{C}$, et al. Oral antidiabetic treatment in type-2 diabetes in the elderly: balancing the need for glucose control and the risk of hypoglycemia. Cardiovasc Diabetol. 2012;11:122.

18. Johnston SS, Conner C, Aagren M, et al. Evidence linking hypoglycemic events to an increased risk of acute cardiovascular events in patients with type 2 diabetes. Diabetes Care. 2011;34:1164-70.

19. Johnston SS, Conner C, Aagren M, et al. Association between hypoglycaemic events and fall-related fractures in Medicare-covered patients with type 2 diabetes. Diabetes Obes Metab. 2012;14:634-43.

20. Laiteerapong N, Karter AJ, Liu JY, et al. Correlates of quality of life in older adults with diabetes: the diabetes \& aging study. Diabetes Care. 2011;34: 1749-53. 
21. Abbatecola AM, Maggi S, Paolisso G. New approaches to treating type 2 diabetes mellitus in the elderly: role of incretin therapies. Drugs Aging. 2008;25:913-25.

22. Punthakee Z, Miller ME, Launer LJ, et al. Poor cognitive function and risk of severe hypoglycemia in type 2 diabetes: post hoc epidemiologic analysis of the ACCORD trial. Diabetes Care. 2012;35:787-93.

23. Minaker KL, Rowe JW, Tonino R, et al. Influence of age on clearance of insulin in man. Diabetes. 1982;31:851-5.

24. Reaven GM, Greenfield MS, Mondon CE, et al. Does insulin removal rate from plasma decline with age? Diabetes. 1982;31:670-3.

25. Chelliah A, Burge MR. Hypoglycaemia in elderly patients with diabetes mellitus: causes and strategies for prevention. Drugs Aging. 2004;21:511-30.

26. Pratley RE, Gilbert M. Clinical management of elderly patients with type 2 diabetes mellitus. Postgrad Med. 2012;124:133-43.

27. VA/DoD clinical practice guideline for the management of diabetes mellitus. Version 4.0. Washington, DC: Veterans Health Administration and Department of Defense; 2010.

28. American Diabetes Association. Standards of medical care in diabetes-2013. Diabetes Care. 2013;36(Suppl 1):S11-66.

29. Lee SJ, Boscardin WJ, Stijacic CI, et al. The risks and benefits of implementing glycemic control guidelines in frail older adults with diabetes mellitus. J Am Geriatr Soc. 2011;59:666-72.

30. Sinclair A, Morley JE, Rodriguez-Manas L, et al. Diabetes mellitus in older people: position statement on behalf of the International Association of Gerontology and Geriatrics (IAGG), the European Diabetes Working Party for Older People (EDWPOP), and the International Task Force of Experts in Diabetes. J Am Med Dir Assoc. 2012;13:497-502.

31. Ross SA, Ekoe JM. Incretin agents in type 2 diabetes. Can Fam Physician. 2010;56:639-48.

32. Raccah D, Miossec P, Esposito V, et al. Efficacy and safety of lixisenatide in elderly ( $>65 \mathrm{yr}$ ) and very elderly ( $>/=75 \mathrm{yr}$ ) patients with type 2 diabetes: an analysis from the GetGoal Phase 3 Program [abstract 972-P]. Diabetes. 2012;61:A248.

33. Umpierrez GE, Meneghini L. Reshaping diabetes care: the fundamental role of DPP-4 inhibitors and
GLP-1 receptor agonists in clinical practice. Endocr Pract. 2013; [Epub ahead of print]. doi:10.4158/ EP12292.RA.

34. Russell S. Incretin-based therapies for type 2 diabetes mellitus: a review of direct comparisons of efficacy, safety and patient satisfaction. Int J Clin Pharm. 2013;35:159-72.

35. Bourdel-Marchasson I, Schweizer A, Dejager S. Incretin therapies in the management of elderly patients with type 2 diabetes mellitus. Hosp Pract (Minneap). 2011;39:7-21.

36. Mathieu C, Bollaerts K. Antihyperglycaemic therapy in elderly patients with type 2 diabetes: potential role of incretin mimetics and DPP-4 inhibitors. Int J Clin Pract. 2007;61:29-37.

37. Byetta [package insert]. San Diego, CA: Amylin Pharmaceuticals, Inc.; 2011.

38. Victoza [package insert]. Princeton, NJ: Novo Nordisk A/S; 2012.

39. Bydureon [package insert]. San Diego, CA: Amylin Pharmaceuticals; 2012.

40. Pencek R, Blickensderfer A, Li Y, et al. Exenatide twice daily: analysis of effectiveness and safety data stratified by age, sex, race, duration of diabetes, and body mass index. Postgrad Med. 2012;124:21-32.

41. Bode BW, Brett J, Falahati A, et al. Comparison of the efficacy and tolerability profile of liraglutide, a once-daily human GLP-1 analog, in patients with type 2 diabetes $>/=65$ and $<65$ years of age: a pooled analysis from phase III studies. Am J Geriatr Pharmacother. 2011;9:423-33.

42. Lowes R. Once-weekly exenatide okayed by FDA for T2DM after long look. 2012. http://www.medscape. com/viewarticle/757664. Accessed 28 Jan 2013.

43. Januvia [package insert]. Whitehouse Station, NJ: Merck \& CO., Inc.; 2012.

44. Onglyza [package insert]. Princeton, NJ: BristolMyers Squibb Company; 2011.

45. Tradjenta [package insert]. Ridgefield, CT: Boehringer Ingelheim International GmbH; 2012.

46. Tucker M. FDA licenses diabetes drug alogliptin, in 3 formulations. 2013. http://wwwmedscape.com/ viewarticle/778235_print. Accessed 28 Jan 2013.

47. Shankar R, Engel S, Xu L, et al. Sitagliptin provides similar glycemic improvement with less hypoglycemia in the elderly with type 2 diabetes mellitus compared to sulfonylurea. Diabetes. 2012;61:A278. 
48. Charbonnel B, Barnett A, Monyak J, et al. Efficacy and safety of saxagliptin in combination with insulin in elderly patients with type 2 diabetes [abstract 1048-P]. Diabetes. 2012;61:A269.

49. Barnett A, Huisman H, Jones R, et al. Efficacy and safety of Linagliptin in elderly patients $(>/=70$ Years) with type 2 diabetes [abstract 1017-P]. Diabetes. 2012;61:A260-1.

50. Iqbal N, Allen E, Donavan M, Ohman P. Long-term safety and tolerability of saxagliptin add-on therapy in elderly patients with type 2 diabetes [abstract 1160-P]. Diabetes. 2012;61:A299.

51. Barzilai N, Guo H, Mahoney EM, et al. Efficacy and tolerability of sitagliptin monotherapy in elderly patients with type 2 diabetes: a randomized, double-blind, placebo-controlled trial. Curr Med Res Opin. 2011;27:1049-58.

52. Janumet XR (sitagliptin and metformin $\mathrm{HCl}$ extended-release) tablets [package insert]. Whitehouse Station, NJ: Merck \& Co., Inc.; 2012.

53. St Onge EL, Miller S, Clements E. Sitagliptin/ metformin (Janumet) as combination therapy in the treatment of type- 2 diabetes mellitus. Pharm Ther. 2012;37:699-708.

54. Pratley RE, McCall T, Fleck PR, et al. Alogliptin use in elderly people: a pooled analysis from phase 2 and 3 studies. J Am Geriatr Soc. 2009;57:2011-9.

55. Clinicaltrials.gov. Study of Sitagliptin in Older Type 2 Diabetics (Clincal trial identifier NCT00305604), 2013. http://clinicaltrials.gov/show/NCT00305604. Accessed 2 Jan 2013.

56. Clinicaltrials.gov. Saxagliptin Compared to Glimepiride in Elderly Type 2 Diabetes Patients, With Inadequate Glycemic Control on Metformin (GENERATION) (Clincal trial identifier NCT01006603), 2013. http://clinicaltrials.gov/ show/NCT01006603. Accessed 26 Jan 2013.

57. Clinicaltrials.gov. Efficacy and Safety of Linagliptin in Elderly Patients With Type 2 Diabetes (Clinical trial identifier: NCT01084005), 2013. http:// clinicaltrials.gov/ct2/show/NCT01084005. Accessed 26 Jan 2013.

58. Clinicaltrials.gov. Efficacy and Safety of Alogliptin Compared to Glipizide in Elderly Diabetics (Clinical trials identifier: NCT00707993), 2013. http:// clinicaltrials.gov/ct2/show/NCT00707993. Accessed 27 Jan 2013.

59. Amori RE, Lau J, Pittas AG. Efficacy and safety of incretin therapy in type 2 diabetes: systematic review and meta-analysis. JAMA. 2007;298:194-206.
60. Arold G, Kupcova V, Thrane M, et al. Insulin degludec has similar pharmacokinetic properties in subjects with hepatic impairment when compared to subjects with normal hepatic function [abstract 1119-P]. Diabetes. 2012;61:A289.

61. European Medicines Agency. Annex 1,2,3 Summary of Product Characteristics Galvus $50 \mathrm{mg}$ tablets. 2012. http://www.ema.europaeu/docs/en_GB/ document_library/EPAR_-_Product_Information/ human/000771/WC500020327.pdf. Accessed 26 Jan 2013.

62. Kiss I, Arold G, Bottcher G, et al. Insulin degludec has similar pharmacokinetic properties in subjects with renal impairment and subjects with normal renal function [abstract 1151-P]. Diabetes. 2012;61:A296-7.

63. Linnebjerg $\mathrm{H}$, Choi $\mathrm{S}$, Lam ECQ, et al. Pharmacokinetics (PK) of the novel, long-acting basal insulin LY2605541 in subjects with varying degrees of renal function [abstract 1148-P]. Diabetes. 2012;61:A296.

64. Babu A. Canagliflozin for the treatment of type 2 diabetes. Drugs Today (Barc). 2013;49:363-76.

65. Demaris KM, White JR. Dapagliflozin, an SGLT2 inhibitor for the treatment of type 2 diabetes. Drugs Today (Barc). 2013;49:289-301.

66. Ferrannini E, Solini A. SGLT2 inhibition in diabetes mellitus: rationale and clinical prospects. Nat Rev Endocrinol. 2012;8:495-502.

67. Nainggolan L. FDA Approves Canagliflozin, a Firstin-Class Diabetes Drug. 2013. http://www. medscape.com/viewarticle/781709. Accessed 1 Aug 2013.

68. Bailey CJ, Gross JL, Hennicken D, et al. Dapagliflozin add-on to metformin in type 2 diabetes inadequately controlled with metformin: a randomized, double-blind, placebo-controlled 102-week trial. BMC Med. 2013;11:43.

69. Cefalu WT, Leiter LA, Yoon KH, et al. Efficacy and safety of canagliflozin versus glimepiride in patients with type 2 diabetes inadequately controlled with metformin (CANTATA-SU): 52 week results from a randomised, double-blind, phase 3 non-inferiority trial. Lancet. 2013; [Epub ahead of print].

70. Bode B, Stenlof K, Sullivan D, et al. Efficacy and safety of canagliflozin treatment in older subjects with type 2 diabetes mellitus: a randomized trial. Hosp Pract (1995). 2013;41:72-84.

71. Nathan DM, Buse JB, Davidson MB, et al. Medical management of hyperglycemia in type 2 diabetes: $\mathrm{a}$ consensus algorithm for the initiation and 
adjustment of therapy: a consensus statement of the American Diabetes Association and the European Association for the Study of Diabetes. Diabetes Care. 2009;32:193-203.

72. Li C, Ford ES, Zhao G, et al. Trends of insulin use among US adults with type 2 diabetes: the behavioral risk factor surveillance system, 1995-2007. J Diabetes Complicat. 2012;26:17-22.

73. Tanwani LK. Insulin therapy in the elderly patient with diabetes. Am J Geriatr Pharmacother. 2011;9:24-36.

74. Zafon C, Creus C. A comparison on insulin regimen treatment of elderly ( $>70$ years) and younger ( $<70$ years) type 2 diabetic patients in actual clinical practice. Acta Diabetol. 2013;50:33-7.

75. Rodbard HW, Jellinger PS, Davidson JA, et al. Statement by an American Association of Clinical Endocrinologists/American College of Endocrinology consensus panel on type 2 diabetes mellitus: an algorithm for glycemic control. Endocr Pract. 2009;15:540-59.

76. Lee $\mathrm{P}$, Chang A, Blaum C, et al. Comparison of safety and efficacy of insulin glargine and neutral protamine hagedorn insulin in older adults with type 2 diabetes mellitus: results from a pooled analysis. J Am Geriatr Soc. 2012;60:51-9.

77. Garber AJ, Clauson P, Pedersen CB, et al. Lower risk of hypoglycemia with insulin detemir than with neutral protamine hagedorn insulin in older persons with type 2 diabetes: a pooled analysis of phase III trials. J Am Geriatr Soc. 2007;55:1735-40.

78. Heise T, Nosek L, Bottcher SG, et al. Ultra-longacting insulin degludec has a flat and stable glucose-lowering effect in type 2 diabetes. Diabetes Obes Metab. 2012;14:944-50.

79. Pandyarajan V, Weiss MA. Design of non-standard insulin analogs for the treatment of diabetes mellitus. Curr Diab Rep. 2012;12:697-704.

80. Jonassen I, Havelund S, Hoeg-Jensen T, et al. Design of the novel protraction mechanism of insulin degludec, an ultra-long-acting basal insulin. Pharm Res. 2012;29:2104-14.

81. Sinha VP, Howey DC, Soon DK, et al. Single-dose pharmacokinetics (PK) and glucodynamics (GD) of the novel, long-acting basal insulin LY2605541 in healthy subjects [abstract 1063-P]. Diabetes. 2012;61:A273.

82. Garber AJ, King AB, Del PS, et al. Insulin degludec, an ultra-longacting basal insulin, versus insulin glargine in basal-bolus treatment with mealtime insulin aspart in type 2 diabetes (BEGIN Basal-Bolus Type 2): a phase 3, randomised, open-label, treat-totarget non-inferiority trial. Lancet. 2012;379:1498-507.

83. Zinman B, Philis-Tsimikas A, Cariou B, et al. Insulin degludec versus insulin glargine in insulin-naive patients with type 2 diabetes: a 1-year, randomized, treat-to-target trial (BEGIN Once Long). Diabetes Care. 2012;35:2464-71.

84. Ratner RE, Gough SCL, Mathieu C, et al. Hypoglycaemia risk with insulin degludec compared with insulin glargine in type 2 and type 1 diabetes: a pre-planned meta-analysis of phase 3 trials. Diabetes Obes Metab. 2013;15:175-84.

85. Gough S, Ratner R, Mathieu C, Del Prato S, Bode B, Mersebach $\mathrm{H}$, et al. Prospectively planned metaanalysis comparing hypoglycaemia rates of insulin degludec with those of insulin glargine in all patients and an elderly ( $>65$ year) subgroup [poster]. European Association for the Study of Diabetes: October 1, 2012; Berlin, Germany.

86. Bergenstal RM, Rosenstock J, Arakaki RF, et al. A randomized, controlled study of once daily LY2605541, a novel long-acting basal insulin, versus insulin glargine in basal insulin-treated patients with type 2 diabetes. Diabetes Care. 2012;35:2140-7.

87. The DCCT Research Group. Epidemiology of severe hypoglycemia in the diabetes control and complications trial. The DCCT Research Group. Am J Med. 1991;90:450-9.

88. Fidler C, Elmelund CT, Gillard S. Hypoglycemia: an overview of fear of hypoglycemia, quality-of-life, and impact on costs. J Med Econ. 2011;14:646-55.

89. Wild D, von Maltzahn R, Brohan E, et al. A critical review of the literature on fear of hypoglycemia in diabetes: Implications for diabetes management and patient education. Patient Educ Couns. 2007;68:10-5.

90. Nguyen HT, Arcury TA, Grzywacz JG, et al. The association of mental conditions with blood glucose levels in older adults with diabetes. Aging Ment Health. 2012;16:950-7.

91. Tasci I, Naharci MI, Bozoglu E, et al. Cognitive and functional influences of vildagliptin, A DPP-4 inhibitor, added to ongoing metformin therapy in elderly with type 2 diabetes. Endocr Metab Immune Disord Drug Targets 2013; [Epub ahead of print].

92. Nandipati S, Luo X, Schimming C, et al. Cognition in non-demented diabetic older adults. Curr Aging Sci. 2012;5:131-5. 
93. Holstein A, Egberts EH. Risk of hypoglycaemia with oral antidiabetic agents in patients with Type 2 diabetes. Exp Clin Endocrinol Diabetes. 2003;111:405-14.

94. Bodmer M, Meier C, Krahenbuhl S, et al. Metformin, sulfonylureas, or other antidiabetes drugs and the risk of lactic acidosis or hypoglycemia: a nested case-control analysis. Diabetes Care. 2008;31:2086-91.
95. Yki-Jarvinen H. Thiazolidinediones. N Engl J Med. 2004;351:1106-18.

96. Hirshberg B, Raz I. Impact of the U.S. Food and Drug Administration cardiovascular assessment requirements on the development of novel antidiabetes drugs. Diabetes Care. 2011;34:S101-6. 